\title{
ENSACAMENTO DE FRUTOS - UMA ANTIGA E EFICIENTE ESTRATÉGIA DE MANEJO DE PRAGAS NA HORTICULTURA.
}

\author{
Adalton Raga ${ }^{1}$, Leonardo Tambones Galdino \\ ${ }^{1}$ Engenheiro Agrônomo, Pesquisador Científico VI, Instituto Biológico, Campinas, SP. \\ E-mail: adalton.raga@gmail.com \\ 2Engenheiro Agrônomo, Mestre, Programa de Pós-Graduação em Sanidade, \\ Segurança Alimentar e Ambiental no Agronegócio, Instituto Biológico, Campinas, SP. \\ E-mail: leo_tambones@hotmail.com
}

\begin{abstract}
RESUMO
No Brasil, o processo de ensacamento de frutos começou a ser adotado mais intensamente pelos produtores na primeira metade do século passado, visando ao controle de pragas frugívoras, como moscas-das-frutas (Diptera: Tephritidae). Essa técnica se tornou tradicional em culturas como goiaba e pêssego de mesa, através do emprego de sacos de papel manteiga. Mais recentemente, novos materiais estão sendo empregados na confeç̧ão de sacos para proteção de frutos na horticultura, como o TNT (tecido não tecido), ou ainda sacos com camada dupla. Embora a disponibilidade de mão-de-obra seja um fator limitante à sua adoção em grandes áreas, em plantas de grande porte ou muito produtivas, o ensacamento de frutos é uma alternativa viável para a agricultura familiar de produção convencional e orgânica. A sua adoção diminui ou evita o uso de agrotóxicos, com melhorias na saúde humana e ambiental. Algumas culturas e respectivas pragas em que o ensacamento de frutos foi avaliado em pré e pós-colheita foram listadas neste trabalho.
\end{abstract}

PALAVRAS-CHAVE: manejo de pragas, controle mecânico, produção orgânica, inseto. 


\section{ABSTRACT}

\section{FRUIT BAGGING - AN OLD AND EFFICIENT STRATEGY FOR PEST MANAGEMENT IN HORTICULTURE.}

In Brazil, the fruit bagging process began to be adopted more intensively by the growers in the first half of the last century, aiming to control frugivorous pests, such as fruit flies (Diptera: Tephritidae). This technique has become traditional in fruit crops like fresh guava and peach, through the use of butter paper bags. More recently, new materials are being used in the manufacture of fruit protection bags in horticulture, such as NWF (non-woven fabric), or even double layer bags. Although the availability of labor is a limiting factor for its adoption in wide areas, or in large or very productive plants, fruit bagging is a viable alternative for family farms with conventional or organic production. Its adoption decreased or avoid the use of agrochemicals, with improvements in human and environmental health. Some crops and their respective pests in which fruit bagging was evaluated in pre- and post-harvest were listed in this paper.

KEYWORDS: Pest management, mechanical control, organic production, insect.

\section{1 - Introdução}

O aumento do consumo de frutas e vegetais tem um grande impacto na saúde pública (VEER et al., 2000). Estudos epidemiológicos têm demonstrado uma significativa correlação positiva entre a ingestão de frutas e vegetais e a redução da taxa de mortalidade por doenças cardíacas, doenças comuns e outras doenças degenerativas, bem como do processo de envelhecimento (KAUR; KAPOOR, 2001). Um grande número de agentes anticarcinogênicos é encontrado nessas fontes alimentares, incluindo carotenóides, vitaminas $C$ e $E$, selênio, fibra alimentar, glicosinolatos e indóis, isotiocianatos, flavonóides, fenóis, inibidores de protease, esteróis de plantas, compostos de alho e limoneno. Esses compostos têm mecanismos de indução de enzimas de desintoxicação, inibição da formação de nitrosaminas (cancerígenas), fornecimento de substratos para formação de agentes antineoplásicos, diluição e ligação de carcinógenos no trato digestivo, alteração do metabolismo hormonal, efeitos antioxidantes, entre outros (STEINMETZ; POTTER, 1991).

Dentre os efeitos prejudiciais causados pelo uso excessivo de pesticidas estão incluídos a segurança dos trabalhadores e dos alimentos, a contaminação ambiental e o declínio da biodiversidade de agroecossistemas. Portanto, o manejo de insetos-praga tem sido uma das questões mais importantes para os fruticultores (CHOl et al., 2008). 
Os frutos são suscetíveis ao ataque de diversos artrópodos pragas. As pragas causam perdas quantitativas e qualitativas durante os processos de produção, colheita e comercialização. Sabe-se que algumas variedades são mais atacadas que outras da mesma espécie botânica. Dependendo da espécie praga, os ovos podem ser colocados na parte externa ou interna dos frutos e neste caso existe uma dificuldade inicial de avaliação de sintomas e danos.

Mesmo quando a postura é feita na superfície dos frutos, existe uma dificuldade em detectar os ovos devido ao tamanho diminuto dessa fase. Por isso é importante treinar o pessoal envolvido naqueles processos da cadeia produtiva de produtos hortícolas, capacitando-os na identificação das espécies pragas e respectivos estágios do ciclo de vida, bem como na sintomatologia em espécies e variedades hortícolas (RAGA; GALDINO, 2017).

A possibilidade de cultivo sem o uso de inseticidas químicos ou o seu uso em quantidades reduzidas, associada às exigências de alimento seguro por parte dos consumidores tem motivado os produtores a retornarem à prática de ensacar frutas (PASTORI et al. 2017).

A proteção completa das plantas com telas de pano visando a sua proteção contra o ataque de moscas-das-frutas foi a primeira estratégia pensada na fruticultura (HEMPEL, 1901). O ensacamento individual de frutas na árvore com sacos de papel para evitar a oviposição e, assim, produzir frutas livres de moscas-das-frutas mesmo na presença de adultos é um método de controle utilizado em muitos países (VIJAYSEGARAN, 1993) e pode conduzir a uma supressão populacional de moscas-das-frutas (ANSARI et al., 2012).

Os diversos tipos de sacos ou invólucros provocam uma proteção física ao fruto, prevenindo a postura das fêmeas de moscas-das-frutas (ANSARI et al., 2012) e no Brasil a sua utilização tem sido recomendada desde a primeira metade do século passado, em pomares de maçã, pêssego ameixa, maracujá doce e inclusive citros (FONSECA, 1952; MARICONI, 1954; MARICONI; IBA, 1955; GONÇALVES et al. 1958; PUZZI et al., 1963; PIZA JUNIOR; BRAGA, 1970; SAMPAIO; ORLANDO, 1971; RAGA et al., 2006). Com a descoberta dos inseticidas sintéticos, essa técnica foi gradualmente abandonada, sendo mantida nas culturas de pêssego, nêspera e goiaba de mesa, onde apenas a aplicação de inseticidas não previne a infestação por moscas-das-frutas.

$\mathrm{Na}$ fruticultura paulista, o ensacamento visando a proteção dos frutos contra o ataque de moscas-das-frutas é a técnica mais tradicional na cultura de goiaba de mesa, sendo ainda mantida pelos proprietários de origem japonesa. 


\section{2 - Materiais Utilizados na \\ Produção de Sacos para Proteção de Frutos}

Existem diversos materiais que podem ser utilizados no processo de ensacamento de frutos, desde sacos de papel jornal, papel comum (kraft) e manteiga, papel sanfonado, organza, náilon, TNT (tecido não tecido), plásticos de baixa densidade (polietileno), plástico microperfurado e ziploc, entre outros (Figura 1).

Alguns sacos apresentam malha de um dos lados para permitir ventilação (SHLOMO, 2015). A maioria desses materiais está disponível em várias cores, sendo branca a mais comum. No caso de insetos, a cor dos sacos e sacolas pode ter ação de repelência, produzindo um efeito aditivo à barreira física do fruto ensacado. Para moscas-das-frutas, NAVARRO-LLOPIS; VACAS (2014) e PIÑERO et al. (2006) observaram este efeito e descreveram como diferentes cores podem ser atrativas, não despertar interesse ou até mesmo causar repelência.

Mais recentemente foi desenvolvido o plástico biodegradável à base de amido para o ensacamento de frutos, que pode durar até nove meses no campo. Esse material foi testado em mangas nas $\mathrm{Fi}$ lipinas e mostrou resultado semelhante ao saco de papel comum nos seguintes parâmetros: cor da casca, cor da polpa, sólidos solúveis totais e ${ }^{\circ} \mathrm{Brix}$ (TUATES JR.; CAPARINO, 2016). Esse tipo de inovação é altamente desejável para a agricultura sustentável.
O resultado prático do ensacamento depende muito da matéria prima utilizada na fabricação do saco, da espécie e variedade do fruto, do processo de respiração envolvido, das condições climáticas e do custo de aquisição local. A facilitação de trocas gasosas pelo material empregado é uma característica desejável, bem como evitar o acúmulo de água no interior do saco, que favorece a ação de patógenos.

O emprego de sacos de papel kraft marrom em Maçãs Granny Smith na Califórnia evita as infestações da traça Cydia pomonella, aumentando em 30$40 \%$ a produção e protegendo os frutos de queimadura de sol e vento excessivo (BENTLEY; VIVEROS, 1992). Em nêsperas, o ensacamento com papel jornal de folha dupla, além de proteger do ataque de moscas-das-frutas, previne a incidência da anomalia denominada "mancha-arroxeada" (OJIMA et al., 1976). O ensacamento pode auxiliar na diminuição dos níveis de resíduos de pesticidas, restringir a aplicação a algumas semanas durante a frutificação, ou até em alguns casos, eliminar a aplicação de agrotóxicos.

Vários tipos de saco utilizados na proteção de mangas (cv. Alphonso) contra $o$ ataque de pragas e doenças foram testados na India e de modo geral, esses materiais não alteraram significativamente os seguintes parâmetros: sólidos solúveis totais, acidez, ácido ascórbico e $\beta$ caroteno (NAGAHARSHITHA et al., 2014). O ensacamento com papel branco, marrom ou polietileno aumentou 
o teor de vitamina $\mathrm{C}$, tamanho e peso dos frutos de goiaba cv. Swarupkathi (RAHMAN et al., 2018).

A formação de pigmentos em frutas também está relacionada com a luz na faixa de UV - visível. Como os efeitos da luz no desenvolvimento das plantas variam com o comprimento de onda, o uso de materiais com diferentes faixas de luz pode afetar o desenvolvimento e a qualidade de frutas. O ensacamento com sacos de polietileno de baixa densidade produzidos para emitir a faixa de UV ( 280-390 nm) e azul visível (( 425-490 nm) acelera o desenvolvimento e aumenta o peso de mangas cv. Nam Dok Mai (CHONHENCHOB et al., 2011).

\section{3 - Tática de Manejo Integrado de Pragas (MIP)}

Dentre as táticas de MIP disponíveis ao produtor podemos destacar o uso de inseticidas, emprego massivo de agentes de controle biológico, ensacamento de frutos e catação de frutos infestados, dentre outras.

O ensacamento de frutos é uma realidade para várias culturas hortícolas, especialmente na fruticultura (Tabela 1), tendo sido uma prática comumente adotada antes do início do emprego massivo de inseticidas sintéticos (NISHIDA, 1958). É uma estratégia eficiente e viável, principalmente para pequenos produtores que não utilizam inseticidas, prevenindo riscos à saúde do produtor e do consumidor.
No Brasil, o ensacamento é uma prática antiga no controle de moscas-das-frutas (Diptera: Tephritidae). É comumente utilizado nas culturas de goiaba, pêssego, marmelo e nêspera (OJIMA et al., 1976). Mais recentemente vem sendo utilizado em tomateiros visando ao controle de brocas (Lepidoptera) de frutos (JORDÃO; NAKANO, 2000).

No caso de moscas-das-frutas, o ensacamento previne a oviposição e deverá ser realizado quando os frutos alcançarem aproximadamente $2,0 \mathrm{~cm}$ de diâmetro nas seguintes espécies: abiu - Pouteria caimito (NASCIMENTO et al., 2011), ameixa - Prunus salicina (SALLES, 1999), goiaba (GOULD; RAGA, 2002; SOUZA-FILHO et al., 2009) e pêssego (SOUZA-FILHO et al., 2009). Essa técnica tem potencial de uso para insetos pragas de diversas Ordens que se alimentam de polpas e sementes de frutos, incluindo Zaprionus indianus Gupta e Drosophila suzukii Matsumura (Drosophilidae).

$\mathrm{Na}$ cultura da bananeira instalada no Vale do Ribeira (SP), o ensacamento com sacos de polietileno impregnados com inseticidas é uma realidade desde a década de 1970, protegendo os dedos contra o ataque da traça Opogona sacchari (Bojer) (PIGATTI et al., 1979). Atualmente, sacos de polietileno impregnados com os ingredientes ativos bifentrina e clorpirifós tem uso autorizado na bananicultura nacional (MAPA. 2019). Também os sacos de TNT são amplamente utilizados na bananicultura. Em ambos os casos. Os sacos deverão ser instalados logo após o início do desenvolvimento dos cachos. 
O ensacamento promove melhorias no tamanho e/ou qualidade dos frutos nas seguintes espécies: pimentão Capsicum annum (LOPES et al., 2018), banana - Musa spp. (CINTRA, 1975; PIGATTI et al., 1979; SATO et al., 2013), manga - Mangifera indica L. (HOFMAN et al., 1997), Tomate - Solanum lycopersicum L. (LEITE et al., 2014), goiaba-serrana - Acca sellowiana (Berg.) Burret. (DONAZOLO; NODARI, 2010), nêspera Eriobotrya japonica, Lindl. (GRASSi et al., 2011) e uva Vitis spp. (OSTAPIV et al., 2006). Os sacos de papel não impedem a incidência de patógenos em goiabas e tampouco protegem os frutos das injúrias mecânicas pós-colheita (MARTINS et al., 2007).

As sacolas, tanto abertas no fundo como fechadas, não são eficientes no controle da mariposa oriental Grapholita molesta (Busk) em pessegueiros (COELHO et al. 2008). No entanto, sacos de papel pardo e papel manteiga foram eficientes na prevenção da incidência de mosca-das-frutas Anastrepha fraterculus (Wied.) e diminuíram a incidência de podridão parda Monilinia fructicola (Wint) Honey em frutos (KESKE et al., 2010).

O ensacamento dos frutos antecipou a maturação de maçãs com a utilização de embalagem plástica transparente microperfurada, e reduziu a coloração vermelha empregando-se o invólucro de TNT (TEIXEIRA et al., 2011). O ensacamento permite produzir maçãs maiores e mais pesadas, aumentar o índice de amido e antecipar a maturação (SANTOS et al., 2015).
Para frutos de pera de película marrom (ex: cv. Housui e Kousui) são utilizados sacos grandes de papel Kraft de cor marrom, colocados cerca de um mês após o final da floração, quando os frutos possuem diâmetro superior a 2,5 - 3,0 $\mathrm{cm}$ e tem um custo de $\mathrm{R} \$ 0,04$ a $\mathrm{R} \$ 0,06$ por fruto (FAORO, 2003). O pH da polpa de pera japonesa cv. Housui (Pyrus pyrifolia var. culta) aumenta com o uso de sacos duplos de papel parafinado, sacos marrons de papel manteiga parafinado, sacos de papel kraft marrons e sacos de pipoca brancos (FAORO; MONDARDO, 2004).

Por motivos de custos e demanda por mão de obra regional, o ensacamento pode não ser viável economicamente para algumas culturas, especialmente naquelas espécies onde a quantidade de frutos é considerável. Um cuidado especial deve ser tomado em relação aos sacos individuais de TNT usados na fruticultura, pois caso fiquem totalmente expandidos durante o período de amadurecimento, as fêmeas de moscas-das-frutas poderão perfurar o tecido e ovipositar no interior dos frutos. Por isso, recomenda-se que os sacos de qualquer material tenham tamanho acima do tamanho padrão de frutos da variedade, de modo a evitar aderência ou rompimentos da embalagem.

O ensacamento dos frutos pode causar alterações nas características visuais e organolépticas em diversas espécies de vegetais (SHARMA et al., 2014; PASTORI et al. 2017). Essa técnica universal necessita avaliação prévia de sua efi- 
cácia para cada variedade de produtos hortícolas e conduzida nas condições edafo-climáticas locais, devido à possibilidade de causar efeitos indesejáveis na qualidade dos frutos.

Por exemplo, figos ensacados com papel kraft marrom retarda a maturação e desenvolve deficiente coloração nos frutos, enquanto saco de polietileno e papel manteiga melhoram a qualidade dos frutos, melhorando o peso e a coloração (MASARO et al., 2005). Cor e firmeza não foram alteradas em tomates ensacados com sacos de TNT, quando o ensacamento dos frutos foi realizado no estádio florido (T1) ou quando os frutos tinham diâmetro de $1,5 \mathrm{~cm}$ (T2). Acidez titulável, sólidos solúveis totais, carotenóides totais, licopeno e ß-caroteno não apresentaram mudanças quando comparado ao controle (PASTORI et al. 2017).

O ensacamento de cachos de tomate na fase de flor ou quando as frutas apresentam um diâmetro de $1,5 \mathrm{~cm}$ não alterou a qualidade pós-colheita (PASTORI et al., 2017). Cachos de tomate cv. "Valerin", ensacados com TNT foram protegidos do ataque da broca Neoleucinodes elegantalis (Guenée), sendo que o ensacamento de frutos com $1,5 \mathrm{~cm}$ de diâmetro incrementou em $21,5 \%$ a produtividade em relação àqueles não ensacados (FILGUEIRAS, 2016).

MARTINS et al. (2007) verificaram que a incidência de pinta-preta (Guignardia psidii), antracnose (Colletorichum spp.) e podridão de Fusicoccum sp. em goiabas comercializadas no Ceagesp foi significativamente maior nos frutos ensacados $(7,7 \%$ dos frutos) que nos não ensacados (2,1\% dos frutos), enquanto que a incidência de doenças pós-colheita provocadas por patógenos que penetram os frutos de goiaba exclusivamente por ferimentos foi significativamente maior nos frutos não-ensacados $(0,8 \%)$ do que naqueles ensacados $(0,3 \%)$.

O ensacamento de caqui 'Jiro' com sacos de jornal ou papel pardo reduziu a incidência do fungo Schyzothyrium pomi (Mont. \& Fr.) e a coloração da epiderme dos frutos, enquanto o tamanho e teor de sólidos solúveis não foram influenciados pelo ensacamento (BIAGI et al., 2007).

O uso de sacos de polietileno de dupla camada diminui a deposição inicial de inseticidas e fungicidas em frutos, diminuindo de 79 a $82 \%$ a contaminação de maçãs (XU et al., 2018), facilitando a sua adoção em sistemas de manejo integrado e promovendo a segurança alimentar.

O ensacamento de goiabas de mesa promove um aumento considerável da população de cochonilhas brancas Planococcus sp. (Pseudococcidae) nos frutos, pois o saquinho forma uma barreira à ação de inimigos naturais e de inseticidas, causando manchas superficiais e inviabilizando a sua comercialização. O ataque de cochonilhas também é favorecido em frutos ensacados de anonáceas (fruta do conde, atemóia e graviola). Por isso, o fruto deve estar sadio no momento do ensacamento.

De forma geral, são citados omo vantagens do ensacamento dos cachos, 
os seguintes aspectos quantitativos e qualitativos (LICHTEMBERG; LICHTEMBERG, 2011) : a) aumento do peso dos cachos; b) produção de frutos mais longos; c) produção de frutos com maior diâmetro; d) encurtamento do período de floração à colheita; e) produção de frutos com melhor coloração, maior brilho e suavidade da casca; f) redução de danos físicos causados pela abrasão de folhas, deposição de poeira e ação de frio, ventos e de granizo; g) redução de danos de pragas e doenças que depreciam a aparência da casca dos frutos; h) proteção dos cachos contra a presença de ninhos de aves e roedores; e i) proteção contra a deposição de produtos químicos nos cachos, especialmente de óleo mineral e fungicidas quando as pulverizações são feitas com atomizadores costais. Em bananeiras cultivadas no estado do Amazonas, na média das cultivares, as variáveis intervalo entre a emergência da inflorescência e a colheita dos cachos, diâmetro dos frutos, relação polpa/casca, peso da penca, $\mathrm{pH}$, textura dos frutos e sólidos solúveis não foram significativamente influenciados pela prática do ensacamento de cachos com saco plástico perfurado de cor azul. No entanto, o ensacamento diminuiu significativamente a produção da cultivar Prata Zulu (SILVA FILHO; MOREIRA, 2005).

\section{4 - Comentários Finais}

O ensacamento de frutos é uma prática agronômica desejável por ser protetiva ambiental e nutricionalmente, além de favorecer a saúde do trabalhador rural por proporcionar redução no uso de agrotóxicos. O sucesso desta tática é dependente de multifatores que devem ser conhecidos e ajustados para se obter o resultado desejado na proteção do produto final. Embora uma atenção especial deve ser dada à viabilidade econômica da sua adoção, essa estratégia deveria ser incentivada na horticultura familiar.

Devido à escassez de literatura recente sobre a utilização de ensacamento no manejo de pragas hortícolas, pesquisas são necessárias para melhorar a compreensão de fatores como: interferência da coloração nas respostas comportamentais dos diferentes insetos pragas; textura do material em relação à aspectos morfológicos e comportamentais das pragas; adequação de diferentes materiais para cada espécie e cultivar; alinhamento entre necessidades agronômicas e ecológicas, como resistência a fatores ambientais, degradabilidade e possibilidade de reutilização.

Essa tecnologia necessita de inovação, através de ferramentas de biotecnologia e nanotecnologia, agregando novos materiais aos sacos de proteção de frutos, com características de resistência e durabilidade, impregnados com subprodutos vegetais e minerais com ação de repelência a artrópodes e reguladores das trocas gasosas. 


\section{5 - Referências}

ALMEIDA, E. Combate à broca da fruta-do-conde. O Biológico, v. 27, n.3, p. 64, 1961.

ANSARI, M.S.; HASAN, F.; AHMAD, N. Threats to fruit and vegetable crops: fruit flies (Tephritidae) - Ecology, Behaviour, and Management. Journal of Crop Science and Biotechnology, v. 15, n. 3, p. 169 $-188,2012$.

BENTLEY, W.J.; VIVEIROS, M. Brown-bagging 'Grany Smith' apples on trees stops codling moth damage. California Agriculture, v.46, p.30-32, 1992.

BIASI, L.A.; PERESSUTI, R.A.; TELLES, C.A.; ZANETTE, F. MIO, L.L.M. Qualidade de frutos de caqui 'Jiro' ensacados com diferentes embalagens. Semina: Ciências Agrárias, v. 28, n. 2, p. 213-218, 2007.

BORGES. R.T.O. Ensacamento de cachos de tomate visando ao controle das brocas-dos-frutos. 2018. 39 p. Dissertação (Mestrado em Agronomia/Fitotecnia) - Universidade Federal de Uberlândia, Uberlândia, 2018.

BRITO, E.A.; BITTENCOURT, M.A.L.; OLIVEIRA, R.A.; FERRAZ, M.I.F.; MOURA, J.I.L. Efeito do ensacamento e pulverização de frutos de gravioleira, frente à broca-do-fruto das anonáceas, Cerconota anonella (Lepidoptera: Oecophoridae). Agrotrópica, v. 22, n. 3, p. 171 - 176, 2010.

BROGLIO-MICHELETTI, S.M.F.; BERTI-FILHO, E. Controle de Cerconota anonella em pomar de gravioleira. Scientia Agricola, v.57, n.3, p.557-559, 2000.

BUSTILLO, A.E.; PEÑA, J.E. Biology and control of the Annona fruit borer Cerconota anonella (Lepidoptera: Oecophoridae). Fruits, v.47, p.81-84, 1992.

CHOI, S.T.; KANG, S.M.; PARK, C.G. Integrated and organic production: insect pest management. Advances in Horticultural Science, v.2, n. 4, p. 294-300, 2008.

CHONHENCHOB, V.; KAMHANGWONG, D.; KRUENATE, J.; KHONGRAT, K.; TANGCHANTRA, N.; WICHAID, N; SINGH, S.P. Preharvest bagging with wavelength-selective materials enhances development and quality of mango (Mangifera indica L.) cv. Nam Dok Mai. Journal of the Science of Food and Agriculture, v. 91, p. 664-671, 2011.

CINTRA, A.F. Opogona sp. - nova praga da bananicultura. O Biológico, v.41, n. p. 223-231. 1975.

COELHO, L.R.; LEONEL, S.; CROCOMO, W.B.; LABINAS, A.M. Avaliação de diferentes materiais no ensacamento de pêssegos. Revista Brasileira de Fruticultura, v. 30, n. 3, p. 822-826, 2008.

DE GRAAF, J. Developing a Systems Approach for Sternochetus mangiferae (Coleoptera: Curculionidae) in South Africa. Journal of Economic Entomology, v. 103, n. 5, p. 1577-1585, 2010.

DONAZZOLO, J.; NODARI, R. O. Efeito do ensacamento sobre a qualidade de frutos de goiabeiraserrana. In: Seminário: Sistemas de Produção Agropecuária - Ciências Agrárias, Animais e Florestais, 2010, 2010, Dois Vizinhos, PR. Anais do Seminário: Sistemas de Produção Agropecuária - Ciências Agrárias, Animais e Florestais, 2010. Curitiba: Editora da UTFPR, 2010.

FAORO, I.D. Técnica e custo para o ensacamento de frutos de pêra japonesa. Revista Brasileira de Fruticultura, v. 25, n. 2, p. 339-340, 2003.

FAORO, I.D.; MONDARDO, M. Ensacamento de frutos de pereira cv. Housui. Revista Brasileira de Fruticultura, v.26, n.1, p. 86-88, 2004. 
FIALHO, A. Ensacamento de Frutos no Cultivo Orgânico de Tomateiro. Dissertação (Mestrado em Ciências Agrárias, área de concentração em Agroecologia) Universidade Federal de Minas Gerais, Montes Claros, 2018.

FILGUEIRAS, R.M.C.; PASTORI, P.L.; PEREIRA, F.F.; COUTINHO, C.R.; KASSAB, S.O.; BEZERRA, L.C.M. Agronomical indicators and incidence of insect borers of tomato fruits protected with non-woven fabric bags. Ciência Rural, v.47, n.6, e20160278, 2017.

FONSECA. J.P. Controle da "mosca das frutas" nos pomares de citrus. O Biológico, v. 18. n. 10, p. 172-173, 1952.

GHORAI A.K. Avoidance of brinjal fruit borer (Leucinodes orbonalis Guenn.) through eco-friendly physical barriers. Journal of Entomological Research, v. 29, n.2, p. 127-129, 2005.

GONÇALVES, O.; KOBER, E.; VARGAS, E. O controle da mosca das frutas em citrus. A Granja, v. 14, n. 133, p. 50-52, 1958.

GRASSI, A.M.; SCARPARE FILHO., J.A.; CHAGAS, E.A.; PIO, R.; PASQUAL, M.; TIZATO, L.H.G.; CHAGAS, P.C. Qualidade de frutos de cultivares de nespereira em função do ensacamento em diferentes estádios de desenvolvimento. Ciência Rural, v.41, n.2, p.227-229, 2011.

HEMPEL, A. Notas sobre a mosca das fructas. Boletim Agricola, v.2, n.3, p. 162-167, 1901.

HERNANDES, J.L.; BLAIN, G.C.; PEDRO JÚNIOR, M.J. Controle de moscas-das-frutas (Diptera: Tephritidae) em cultivo orgânico de ameixa pelo ensacamento dos frutos com diferentes materiais. Revista Brasileira de Fruticultura, v. 35, n. 4, p. 1209-1213, 2013.

HOFMAN, P.J.; SMITH, L. G.; JOYCE, D.C.; JOHNSON, G.I.; MEIBURG, G.F. Bagging of mango (Mangifera indica cv. 'Keitt') fruit influences fruit quality and mineral composition. Postharvest Biology and Technology, v. 12, p. 83-91, 1997.

ISABIRYE, B.E.; NANKINGA, C.K.; MAYAMBA, A.; AKOL, A.M.; RWOMUSHANA, I. Integrated Management of Fruit Flies - Case Studies from Uganda. p. 497- 515. In EKESI et al. (eds.), Fruit Fly Research and Development in Africa - Towards a Sustainable Management Strategy to Improve Horticulture. Springer International Publishing, 2017.

JORDÃO, A.L.; NAKANO, O. Controle de lagartas dos frutos do tomateiro pelo ensacamento das pencas. Anais da Sociedade Entomológica do Brasil, v. 29, n. 4, p. 773 782, 2000.

KAUR, C.; KAPOOR, H.C. Review Antioxidants in fruits and vegetables - the millennium's health. International Journal of Food Science and Technology, v. 36, p. 703 - 725, 2001.

LEITE, G.L.D.; FIALHO, A.; ZANUNCIO, J.C.; REIS JÚNIOR, R. COSTA, C.A. Bagging tomato fruits: a viable and economical method of preventing diseases and insect damage in organic production. Florida Entomologist, v. 97, n.1, p. 50-60, 2014.

LICHTEMBERG, L.A.; LICHTEMBERG, P.S.F. Avanços na Bananicultura Brasileira. Revista Brasileira de Fruticultura, Volume Especial, p. 29-36, 2011.

LOPES, S.M.; ALCANTA RA, E.; REZENDE, R.M.; FREITAS, A.S. avaliação de frutos de pimentão submetidos ao ensacamento no cultivo orgânico. Revista da Universidade Vale do Rio Verde, v. 16, n. 1, p. 1-11, 2018.

LOURENÇÃO, A.L.; MARTINS, F.P.; NÁVIA, D. Insetos e Ácaros da Videira. In POMMER, C.V. Uva: Tecnologia de Produção. Pós-colheita. Mercado. Editora Cinco Continentes. 2003. 778p. 
MAGAGULA, C.N.; NZIMA, B.A. National response to a new incursion by an invasive fruit fly pest species, Bactrocera dorsalis (Diptera: Tephritidae) in northern Swaziland. International Journal of Agriculture and Environmental Research, v. 3, n. 03, 2017.

MAPA - Ministério da Agricultura, Pecuária e Abastecimento. Sistema de agrotóxicos fitossanitários. Disponível em http://agrofit.agricultura.gov.br/agrofit_cons/ principal_agrofit_cons. Acesso em: 25 jun 2019.

MARICONI, F.A.M. Frutos de maracujá danificados por insetos. O Biológico, v. 20, n.1, p. 14. 1954.

MARICONI, F.A.M.; IBA, S. A mosca do mediterrâneo. O Biológico, v. 21, n.2, p. 17-32, 1955.

MARTINS, M.C.; AMORIM, L.; LOURENÇO, S.A.; GUTIERREZ, A.S.D.; WATANABE, H.S. Incidência de danos pós-colheita em goiabas no mercado atacadista de São Paulo e sua relação com a prática de ensacamento dos frutos. Revista Brasileira de Fruticultura, v. 29, n. 2, p. 245-248, 2007.

MAZARO, S.M.; GOUVEAA, A.; CITADIN, I.; DANNER, M.A. Ensacamento de figos cv. "Roxo de Valinhos". Scientia Agraria, v.6, n.1-2, p.59-63, 2005.

MONDAL, C.K.; GARAIN, P.K.; MAITRA, N.J.; MAJI, A. Biofriendly management of guava fruit fly (Bactrocera correta Bezzi) through wrapping technique. Journal of Applied and Natural Science, v.7, n. 1, p. $358-363,2015$.

NAGAHARSHITHA, D.; KHOPKAR, R.R.; HALDANKAR, P.M.; HALDAVANEKA, P.C.; PARULEKAR. Y.R. Effect of bagging on chemical properties of mango (Mangifera indica L.) cv. Alphonso. Agrotechnology, v. 3, n. 1, p. 1-4, 2014.

NASCIMENTO, W.M.O.; MÜLLER, C.H.; ARAÚJO, C.S.; FLORES. B.C. Ensacamento de frutos de abiu visando à proteção contra o ataque da mosca-das-frutas. Revista Brasileira de Fruticultura, v. 33, n. 1 , p. $48-52,2011$.

NAVARRO-LLOPIS, V.; VACAS, S. Mass Trapping for Fruit Fly Control. p. 513-555In: SHELLY, T. et al. (Ed.). Trapping and the Detection, Control, and Regulation of Tephritid Fruit Flies - Lures, Area-Wide Programs, and Trade Implications. Dordrecht: Springer Netherlands, 2014.

NISHIDA, T. Pollination of the passion fruit in Hawaii. Journal of Economic Entomology, v. 51, n.2, p. 146-149, 1958.

OJIMA, M.; RIGITANO, O.; SIMÃO. S.; IGUE. T. Influência da época de ensacamento da nêspera, na incidência da "mancha-arroxeada" e das moscas-das-frutas. Bragantia, v. 35, n.1, p. 67-69, 1976.

OJIMA, M.; CAMPO-DALL ORTO, F. A.; BARBOSA, W.; MARTINS, F. P.; SANTOS, R. R. Cultura da nespereira. Campinas: IAC, 1999. 36 p. (Boletim Técnico no 185).

OSTAPIV, F. N.; MAZARO, S. M.; DONAZZOLO, J.; CITADIN, I.; LINK, M.; GOUVÊA, A. Influência do ensacamento sobre a qualidade da uva 'Vênus'. Synergismus Scyentifica, v. 1, p. 64-69, 2006.

PASTORI, P.L.; FILGUEIRAS, R.M.C.; OSTER, A.H.; BARBOSA, M.G.; SILVEIRA, M.R.S.; PAIVA, L.G.G. Postharvest quality of tomato fruits bagged with nonwoven fabric (TNT). Revista Colombiana de Ciencias Hortícolas, v. 11, n. 1, p. 80-88, 2017.

PEÑA, J.E.; NADEL, H.; BARBOSA-PEREIRA, M.; SMITH. D. Pollinators and pests of Annona species. p. 197-221. In PEÑA, J.E.; SHARP, J.L.; WYSOKI, M. (eds.), Tropical fruit pests and pollinators. CAB Inter. Pub., United Kingdom, 2002.

PIGATTI, A.; OLIVEIRA, D.A.; ALMEIDA. P.R.; CINTRA. A.F. Emprego de sacos de polietileno para proteção de cachos de banana no controle da "traça" - Opogona sacchari (Bojer, 1856) $(=0$. subcervinella Walker 1863) - Lepidoptera: Lyonetidae. O Biológico, v. 45, n. 11-12, p. 285-288, 1979. 
PIÑERO, J. C.; JÁCOME, I.; VARGAS, R.; PROKOPY, R. J. Response of female melon fly, Bactrocera cucurbitae, to host-associated visual and olfactory stimuli. Entomologia Experimentalis et Applicata. v. 121, p. 261-269, 2006.

PIZZA JUNIOR. C.T.; BRAGA, F.G. Cultura do Pessegueiro. Boletim Técnico $n^{\circ} 29$. Coordenadoria de Assistência Técnica Integral. Campinas -SP, 1970. 144p.

PUZZI, D.; RIGITANO, O.; ORLANDO, A. Combate às "moscas das frutas" em pêssegos com pulverização de Lebaycid. O Biológico, v. 29, n. n.9. p. 189-190. 1963.

RAGA, A.; SOUZA FILHO, M.F.; RODRIGUES NETTO. S.M. Pragas do Maracujazeiro. In Aspectos Fitossanitários do Maracujazeiro (Boletim Técnico 14). NOGUEIRA. E.M.C.; FERRARI. J.T. (Coord.). Instituto Biológico.2006. 81p.

RAGA, A.; GALDINO, L.T. Sintomatologia do Ataque de Moscas-das-frutas (Diptera: Tephritidae) em Citros. São Paulo: Instituto Biológico, 2017 (Documento Técnico $n^{\circ} 33$ ), 17p. Disponível em: <http:// www.biologico.sp.gov.br/uploads/dt/089dc7da-722e-4123-ae87-8bfd9f1f519a.pdf. Acesso em 15 jan 2019.

RAHMAN, M.; HOSSAIN, M.; RAHIM, A.; RUBEL1, H.K.; ISLAM, Z. Effect of pre-harvest fruit bagging on post-harvest quality of guava cv. Swarupkathi. Fundamental and Applied Agriculture, v. 3, n.1, p. 363-371, 2018.

SALLES, L.A. Ocorrência precoce da mosca das frutas em ameixas. Ciência Rural, v. 29, n. 2, p. 349-350, 1999.

SAMPAIO, A.S.; ORLANDO, A. Seleção de novos praguicidas no combate à "mosca sul americana". Anastrepha fratercula (Wied.). em goiaba. O Biológico, v. 37, n. 3, p. 62 - 65, 1971.

SANTOS, J.P.; HICKEL, E.R; ARGENTA, L.C. Efeito do ensacamento na qualidade de maçãs em diferentes estádios de desenvolvimento. Revista Brasileira de Fruticultura, v. 26, n. 1, p. 86-88, 2004.

SANTOS, H. A. A.; BOHNEBERGER, A.L.; BOFF, P. Ensacamento de frutos; viabilização de produção orgânica da goiabeira-serrana. In: VII Congresso Brasileiro de Agroecologia, 2011, Fortaleza. 2011, v. 6, n.2, p. $1-5$.

SATO, M. E.; MINEIRO, J.L.C.; RAGA, A. Tripes e ácaros em bananeiras (Musa sp.). In: NOGUEIRA, E.M.C.; ALMEIDA, I.M.G.; FERRARI, J.T.; BERIAM, L.O.S. (Org.). Bananicultura: Manejo Fitossanitário e Aspectos Econômicos e Sociais da Cultura. 1ed. São Paulo: Instituto Biológico, 2013, v. 1, p. 165-171.

SARKER, D.; RAHMAN, M.M.; BARMAN, J.C. Efficacy of different bagging materials for the control of mango fruit fly. Bangladesh Journal of Agricultural Research, 34, n. 1, p. 165-168, 2009.

SAUCO, V.S.; MENINI, U.G.; TINDALL, H.D. Carambola cultivation. Rome: FAO, 1993. 74p.

SHLOMO, M. Efficiency of bagging pomegranate fruits. Acta Horticulturae, v. 1089, p. 485-488, 2015.

SILVA, F.F.; MEIRELLES, R.N.; SOGLIO, F.K.; REDAELLI, L.R. Comparação de métodos de controle de moscas-das-frutas (Diptera: Tephritidae) na produção orgânica de citros. Revista da Faculdade de Zootecnia, Veterinária e Agronomia, v.14, n.1, p. 36-52, 2007.

SILVA FILHO, L.P.; MOREIRA, A. Ensacamento de cachos na produção, maturação e qualidade dos frutos de bananeiras cultivadas no Estado do Amazonas. Acta Amazonica, v. 35, n. 4, p. 407 - 412, 2005. 
SOUZA, J.C.; RAGA, A.; SOUZA, M.A. Pragas da goiabeira. Boletim Técnico $n^{\circ} 71$. Epamig. Belo Horizonte. 2003. 60p.

SOUZA-FILHO, M.F. ; RAGA, A. ; AZEVEDO FILHO, J.A.; STRIKIS, P.C.; GUIMARÃES, J.A.; ZUCCHI, R.A. Diversity and seasonality of fruit flies (Diptera: Tephritidae and Lonchaeidae) and their parasitoids (Hymenoptera: Braconidae and Figitidae) in orchards of guava, loquat and peach. Brazilian Journal of Biology, v. 69, p. 31-40, 2009.

STEINMETZ, K.A.; POTTER, J.D. Vegetables, fruit, and cancer. II. Mechanisms. Cancer Causes and Control, v. 2, p. $427-442,1991$.

TAVARES, A.M.; SOUZA, A.G.C.; NUNES, A.B. Controle mecânico da broca-do-fruto do cupuaçuzeiro, Conotrachelus sp. (Coleoptera: Curculionidae). Embrapa Amazônia Oriental. Comunicado Técnico 15. 2002, 3p.

TEIXEIRA, R.; BOFF, M.I.C.; AMARANTE, C.V.T.; STEFFENS, C.A.; BOFF, P. Efeito do ensacamento dos frutos no controle de pragas e doenças e na qualidade e maturação de maçãs 'Fuji Suprema'. Bragantia, v. 70, n. 3, p.688-695, 2011.

TUATES JR., A.M.; CAPARINO, O.A. Development of biodegradable plastic as mango fruit bag. International Journal on Advanced Science and Engineering, v.6, n.5, p. 799-806, 2016.

VEER, P.V.; JANSEN, M.C.J.; KLERK, M.; KOK, F.J. Fruits and vegetables in the prevention of cancer and cardiovascular disease. Public Health Nutrition, v. 3, n. 1, p. 103-107, 2000.

VENDRUSCOLO, E.P.; LEAL, L.R.; SOUSA E SILVA, B.C.; CAMPOS, L.F.C.; MARTINS, A.P.B.; SELEGUINI, A. Material evaluation for bagging of cantaloupe melons. Ciência Rural, v.47: 08, e20161016, 2017.

VIJAYSEGARAN, S. Control of Fruit Flies in the Tropical Regions of Asia. p. 455 - 463. In Fruit Flies: Biology and Management. ALUJA, M.; LIEDO, P. (eds.). SpringerVerlag New York, Inc., 1993.

XIA, Y.; HUANG, J.; JIANG, F.; HE, J.; PAN, X.; LIN, X.; HU, H.; FAN, G., ZHU, S.; HOU, B.; OUYANG, $G$. The effectiveness of fruit bagging and culling for risk mitigation of fruit flies affecting citrus in China: a preliminary report. Florida Entomologist, v. 102, n. 1, p.79-84, 2019.

XU, G.; NIE, J.; WU, Y.; YAN, Z.; YE, M. The effects of fruit bagging on residue behavior and dietary risk for four pesticides in apple. Scientific Reports, v. 8, p. 14348, 2018.

\section{Recebido em: 30/07/2019}

\section{Aprovado em: 04/10/2019}



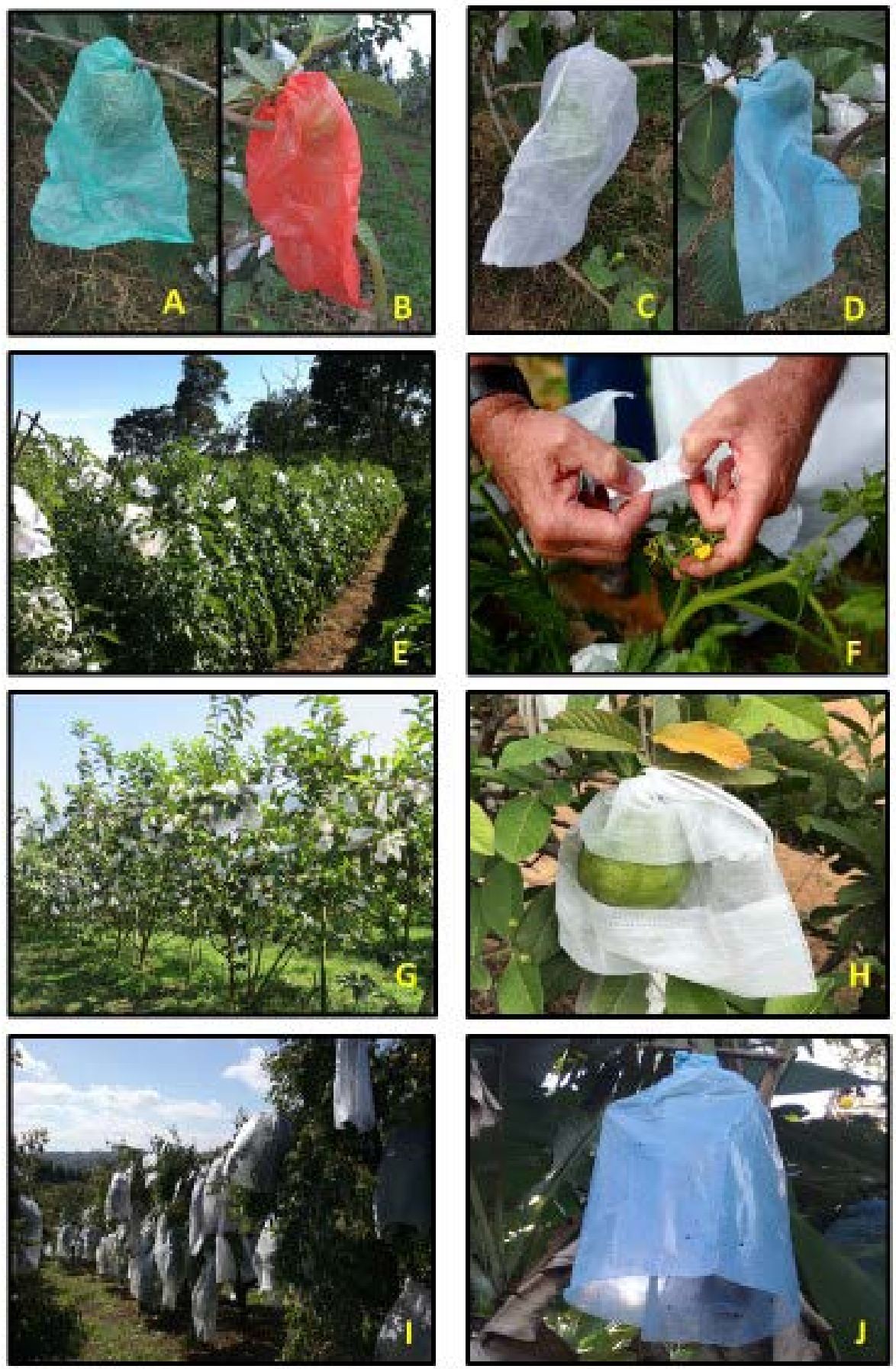

Figura 1. Sacos de TNT de diferentes cores (A,B,C,D); tomate envarado ensacado com TNT (E); ensacamento após o pegamento da flor do tomateiro $(F)$; goiabas ensacadas com papel manteiga (G; H); carambolas ensacadas com TNT (I); cacho de banana ensacado com plástico perfurado (J). (Autoria das fotos E e F: Mario Mezzedimi). 


\section{Tabela 1.}

\begin{tabular}{|c|c|c|c|}
\hline Fruto (Espécie) & Praga ou Grupo de pragas & Tipo de saco & Referência \\
\hline Ameixa (Prunus salicina) & $\begin{array}{l}\text { Ceratitis capitata (Wied.) e } \\
\text { Anastrepha fraterculus (Wied.) }\end{array}$ & TNT & Hernandes et al., 2013 \\
\hline Anonáceas (Annona spp.) & Bephratelloides spp. & - & Peña et al., 2002 \\
\hline \multirow[t]{2}{*}{ Banana (Musa spp.) } & Opogona sacchari (Bojer) & Polietileno azul & Pigatti et al., 1979 \\
\hline & Tripes (Thysanoptera) & Polietileno azul & Sato et al., 2013 \\
\hline Figo (Ficus carica L.) & Diptera & $\begin{array}{l}\text { Polietileno e papel } \\
\text { manteiga }\end{array}$ & Mazaro et al., 2003 \\
\hline $\begin{array}{l}\text { Fruta-do-conde (Annona } \\
\text { squamosa, L.) }\end{array}$ & Cerconota anonella Sepp & Papel & Almeida, 1961 \\
\hline $\begin{array}{l}\text { Cupuaçú (Theobroma grandiflorum } \\
\text { Schum.) }\end{array}$ & Conotrachelus sp. & Plástico & Tavares et al., 2002 \\
\hline \multirow{3}{*}{ Goiaba (Psidium guajava L.) } & C.capitata, Anastrepha spp. & $\begin{array}{l}\text { Plástico e papel } \\
\text { manteiga }\end{array}$ & Souza-Filho et al. 2009 \\
\hline & Bactrocera correta Bezzi & $\begin{array}{l}\text { Plástico } \\
\text { prolipropileno }\end{array}$ & Mondal et al., 2015 \\
\hline & Conotrachelus psidii Marshall & Papel & Souza et al., 2003 \\
\hline Goiaba-serrana (Acca sellowiana) & C. psidii & $\begin{array}{l}\text { Plástico } \\
\text { microperfurado }\end{array}$ & Santos et al., 2011 \\
\hline \multirow{3}{*}{ Graviola (Annona muricata L.) } & Cerconota anonella Sepp & TNT vermelho & Brito, 2010 \\
\hline & C. anonella & $\begin{array}{l}\text { Plástico } \\
\text { microperfurado }\end{array}$ & $\begin{array}{l}\text { Broglio-Micheletti; Berti- } \\
\text { Filho, } 2000\end{array}$ \\
\hline & C. anonella & Plástico & Bustillo; Peña, 1992 \\
\hline $\begin{array}{l}\text { Laranja (Citrus sinensis) } \\
\text { Tangerina Murcot (C. sinensis x } C \text {. } \\
\text { reticulata) }\end{array}$ & A. fraterculus & $\begin{array}{l}\text { Papel sanfonado } \\
\text { pardo }\end{array}$ & Silva et al., 2007 \\
\hline \multirow[b]{2}{*}{ Maçã (Malus sp.) } & Cydia pomonella (Linnaeus) & Papel marrom & Bentley; Viveros. 1992 \\
\hline & $\begin{array}{l}\text { Anastrepha fraterculus (Wied.), } \\
\text { Grapholita molesta (Busck, 1916), } \\
\text { Bonagota salubricola (Meyrick) }\end{array}$ & $\begin{array}{l}\text { Plástico } \\
\text { microperfurado e } \\
\text { saco de TNT }\end{array}$ & Teixeira et al., 2011 \\
\hline
\end{tabular}




\begin{tabular}{|c|c|c|c|}
\hline Fruto (Espécie) & Praga ou Grupo de pragas & Tipo de saco & Referência \\
\hline \multirow{3}{*}{ Manga (Mangifera indica L.) } & Bactrocera dorsalis Hendel & Papel marrom & $\begin{array}{l}\text { Sarker et al., 2009; } \\
\text { Magagula \& Nzima, } 2017\end{array}$ \\
\hline & Bactrocera spp. & Papel & Isabirye et al., 2017 \\
\hline & Sternochetus mangiferae (F.) & Plástico & Graaf, 2010 \\
\hline Maracujá (Passion spp.) & B. dorsalis & $\begin{array}{l}\text { Papel branco, } \\
\text { papel journal }\end{array}$ & Saúco et al., 1993 \\
\hline Nêspera (Eriobotrya japonica Lindl.) & Moscas-das-frutas & $\begin{array}{l}\text { Papel jornal de } \\
\text { paredes duplas }\end{array}$ & Ojima et al., 1976 \\
\hline $\begin{array}{l}\text { Pêra Japonesa Pyrus pyrifolia } \\
\text { (Burm. f.) Nakai }\end{array}$ & A. fraterculus & Papel kraft & Faoro, 2003 \\
\hline Romã (Punica granatum L.) & Insetos em geral & Papel e malha & Shlomo, 2015 \\
\hline \multirow{4}{*}{ Tomate (Solanum lycopersicum L.) } & Tuta absoluta (Meyrick) & TNT & Leite et al., 2014 \\
\hline & $\begin{array}{l}\text { Neoleucinodes elegantalis } \\
\text { (Guenée), Helicoverpa zea } \\
\text { (Boddie) }\end{array}$ & Papel manteiga & Jordão; Nakano, 2000 \\
\hline & T. absoluta, N. elegantalis e H. zea & TNT e organza & Fialho, 2009 \\
\hline & $\begin{array}{l}\text { T. absoluta, N. elegantalis. } \\
\text { Phthorimaea operculella (Zeller) e } \\
\text { H. zea }\end{array}$ & Tule e organza & Borges 2008 \\
\hline $\begin{array}{l}\text { Beringela } \\
\text { (Solanum } \\
\text { melongena L.). }\end{array}$ & Leucinodes orbonalis Guenn. & $\begin{array}{l}\text { Saco de } \\
\text { polietileno } \\
\text { transparente }\end{array}$ & Ghorai, 2005 \\
\hline Uva (Vitis spp.) & Ceratitis capitata (Wied.) & Sacos variados & Lourenção et al., 2003 \\
\hline $\begin{array}{l}\text { Pomelo (Citrus } \\
\text { maxima (Burm. } \\
\text { fil.) Osbeck }\end{array}$ & Bactrocera dorsalis (Hendel) & Não definido & Chia et al., 2019 \\
\hline $\begin{array}{l}\text { Melão } \\
\text { (Cucumis melo } \\
\text { var. } \\
\text { cantalupensis } \\
\text { Naud) }\end{array}$ & Diaphania spp. & Saco de TNT & Vendruscolo et al., 2017 \\
\hline
\end{tabular}

